\title{
The differential diagnosis between andropause and erectile dysfunction
}

\author{
Gerald Brock MD FRCSC
}

\section{G Brock. The differential diagnosis between andropause} and erectile dysfunction. J Sex Reprod Med 2001;1(2):81-84.

The present review identifies elements that are essential to the treatment of erectile dysfunction and andropause; the factors that should be gleaned from patient history and physical findings to help to define which disorder is most likely to be the cause of a patient's concerns; and what treatment options exist. The major diagnostic approaches that should be undertaken for the comprehensive evaluation of the male patient with androgen deficiency of the aging male or erectile dysfunction are highlighted. Determining which condition is present is essential for optimal patient management (12).

Key Words: Aging process; Hormonal deficiences; Impact scale of erectile dysfunction; Intensity scale of erectile dysfunction; Primary erectile disorders; Secondary erectile disorders

\section{Diagnostic différentiel entre andropause et dysérection}

RÉSUMÉ : Le présent article porte sur trois points : les éléments essentiels au traitement de la dysérection et de l'andropause; les facteurs que les antécédents personnels et l'examen physique devraient permettre de recueillir pour définir le trouble le plus susceptible d'être en cause; les traitements possibles. On y décrira les principales approches diagnostiques visant à procéder à l'évaluation globale du patient atteint soit d'un déficit en androgènes lié au vieillissement, soit de dysérection. Il est essentiel de savoir de quel trouble il s'agit afin de mettre en œuvre le meilleur traitement possible (12).
Tn the past decade, there have been important advances in Ithe understanding of male sexual physiology (1). Innovative research involving human volunteers and animal models evaluating the neurophysiological pathways essential for erection has led to significant clinical progress in this field $(2,3)$. Historically, treatment of sexual dysfunction (erectile disorders, ejaculatory and desire deficits) has largely been delegated to symptom improvement. The ability to localize specific targets of dysfunction is improving as research continues to expand knowledge of normal physiology. Research initiatives aimed at identifying and purifying agents able to selectively stimulate the erectile pathway in men with isolat- ed defects are ongoing and show great promise (4-8). Ironically, as the therapeutic options available to treat erectile dysfunction (ED) expand, the limiting factor in physicians' ability to effectively reach the millions of affected North American men may be the stigma associated with impotence.

Traditionally, ED has been an uncomfortable subject for polite conversation, let alone conversation between physicians and patients. Medical school curricula have left the field of sexual medicine largely uncovered at most North American universities. Armed with effective, minimally invasive oral therapy, as well as an impressive information campaign about ED targeted at the public and delivered by 
TABLE 1

Symptoms and/or events usually associated with erectile dysfunction or andropause

\begin{tabular}{ll}
\hline Erectile dysfunction & Andropause \\
\hline Gradual onset & Gradual onset \\
Predominant erectile problems & Erections usually fine \\
Often associated vascular problems & Decreased sexual desire \\
Sexual desire variable & Low energy \\
Age variable & Impaired visual spatial skills \\
Normal energy & Depression-like symptoms \\
Usually responsive to erectile & Usually biochemical evidence \\
dysfunction medications & of hypoandrogenism \\
Hormone levels usually normal & \\
Hormone replacement therapy & \\
usually not able to completely & \\
restore erectile function & \\
\hline
\end{tabular}

well respected political figures, public perception of ED seems to be shifting in a positive direction (9). Improved community perception of this problem may translate into greater openness for treatment.

In contrast to ED, andropause, or androgen deficiency in the aging male (ADAM) as described by Jean Mailhot in the present issue (pages 75 to 77), has been studied to an even smaller degree. This more recently described symptom complex has fewer concrete end points and, to many, remains a constellation of symptoms that is not yet defined fully $(10,11)$. It truly is a term of convenience, simply describing a clinical scenario that occurs in a large number of aging men with low or low to normal levels of testosterone, inadequate energy and desire, and malaise-like complaints.

The good news is that times are changing. Medical schools and departments of continuing medical education across Canada and the United States are now recognizing the need to educate their constituents about ED and ADAM, two important male symptom complexes. Important advances in therapeutics, and in the understanding of both of these conditions are leading to greater public awareness and patient satisfaction after treatment.

In this brief review, elements essential to the treatment of ED and andropause are identified, as are the factors that should be gleaned from patient history and physical findings to help to define which disorder is most likely to be the cause of the patient's concerns, and what treatment options exist. The major diagnostic approaches that should be undertaken for the comprehensive evaluation of the male patient with ADAM and/or ED are highlighted. These are important issues, because determining which condition is present is essential for optimal patient management (12).

\section{ARE THEY REALLY THE SAME CONDITION?}

As depicted in Table 1, ED and ADAM are distinct clinical conditions with significant symptom overlap. In the author's view, the early determination of sexual desire should serve as an important decision point along the treatment algorithm.

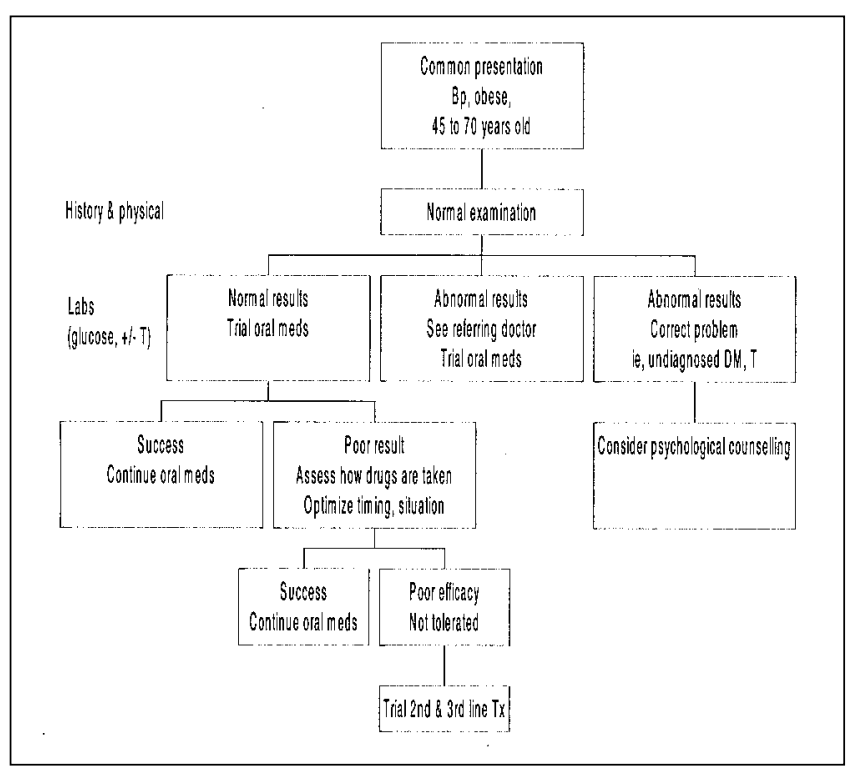

Figure 1) Possible issues secondary to history and physical examination of an individual experiencing erectile dysfunction. Bp High blood pressure; DM Diabetes mellitus; T Thyroid disorder; Tx Treatment

Once a complete history is obtained (Figure 1) by focusing on desire, morning erections, length of time since the beginning of the complaint and associated areas of concern, a clear idea of the dysfunction is usually apparent. Determining what aspect of erectile function is lacking (degree of rigidity, difficulty in obtaining or maintaining an erection, etc) can help to differentiate between the two conditions. Alternatively, most men with ADAM report collateral issues that dominate their concerns, including a lack of energy, loss of visual spatial skills or general fatigue, often of chronic or subchronic duration.

In truth, there often is confusion between the two conditions, because many men with 'pure' ED develop secondary low desire states. Men who suffer from ED frequently do not want to keep trying to have sexual contact when they anticipate inadequate results. Similarly, men with ADAM may not be sexually active at all. When completing the standard Sexual Health Inventory for Men (a validated questionnaire), failure may occur from inactivity alone. Additionally, diagnostic testing using devices such as Rigiscan (Timm Medical Technologies, Inc, USA) may have indeterminate results because of impaired nocturnal penile tumescence events, a recognized testosterone effect.

This overlap in presentation style and the general degree of culturally based discomfort in broaching the subject of sexual performance can lead to a diagnostic dilemma. The clinician needs to be aware of these issues and focus on the symptom complex to most effectively manage this situation, because the therapeutic approach is different for erectile dysfunction and andropause.

\section{WHAT ARE THESE CONDITIONS?}

Alterations in the biochemical profiles of men and women with senescence occur at vastly different rates, with significantly different physiological outcomes. At 
menopause, women undergo a rapid loss of hormones because of ovarian failure, lose the ability to reproduce and abruptly stop their monthly cycles, usually over a period of months. This effect is predictable on an individual basis, and occurs between the fourth and fifth decades of life in the vast majority of women. Only recently have the alterations in hormonal profiles in men been recognized and their physiological consequences appreciated (13). This occured most likely, in part, because the changes that most men undergo are much more gradual and do not have easily definable end points as with menstruation; also, they may not occur in all men. Additionally, the wide ranging physiological effects of testosterone and the threshold levels essential for its function still are not understood fully.

The symptom complex known as andropause or ADAM remains controversial, and a more inclusive descriptive term may be needed; however, few health care professional interested in this field would question the validity or importance of this clinical entity $(14,15)$. In contrast to andropause, ED has very clear and objective validated measures; just like andropause, it is highly prevalent within North American society but has specific treatment algorithms and approaches.

\section{The approach}

ADAM is generally characterized by slow onset, often insidious in nature. The essential features that the author relies on for a diagnosis are:

- decreased sexual desire;

- reduced frequency and/or quality of nocturnal erections;

- mood changes (these can vary from mild to severe and may simulate depression);

- reduced muscle mass;

- reduced body hair;

- reduced bone density; and

- increased visceral fat.

The diagnostic challenge of this syndrome is its variability with the aging process, symptom expression and degree of inconvenience experienced by the patient. Many of these changes can be regarded as physiological with advancing age, and many clinicians and patients accept them as such. In the author's view, men who present with this constellation of symptoms or a portion of them deserve to be evaluated for this potentially reversible condition. A high level of suspicion for ADAM is necessary when screening men with the above complaints.

In most men older than age 50 years, a $1 \%$ decrease in serum testosterone concentrations per year is expected. In

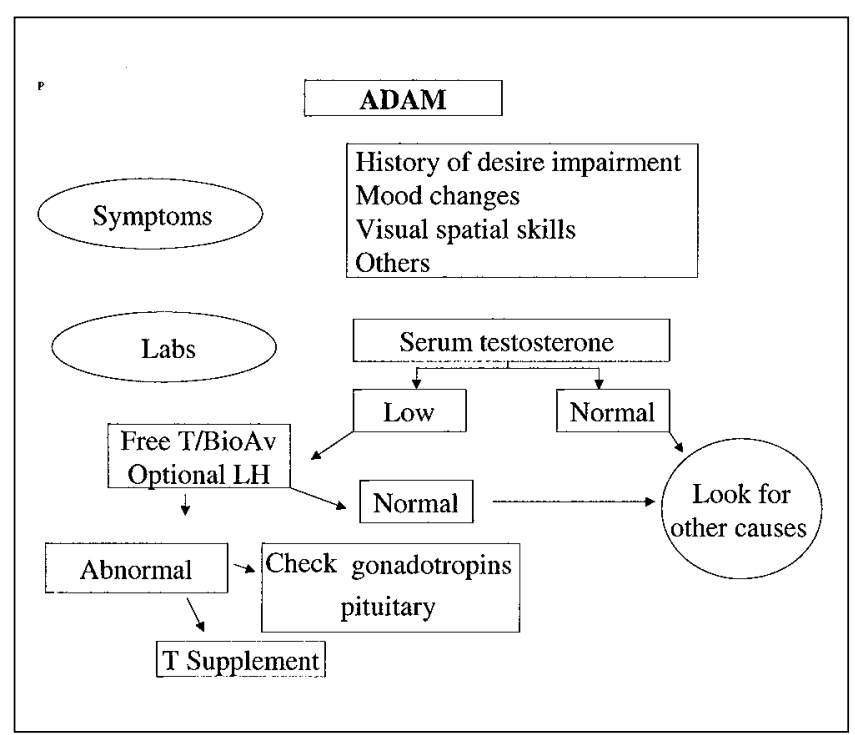

Figure 2) Investigation algorithm for androgen deficiency in the aging male (ADAM). BioAv Bioavailable testosterone; LH Luteinizing hormone; $T$ Testosterone

contrast, the levels of sex hormone-binding globulin increase with advancing age. These two physiological facts indicate that significant numbers of men older than age 50 years experience relatively low levels of free or bioavailable (free or bound to albumin) testosterone (15). Recent reports have evaluated the role of luteinizing hormone (LH) in helping to define better the men most likely to respond to replacement therapy by measuring the LH response. These data are not yet mature, but elevated LH levels may be another marker of early ADAM (16). In the approach to the problem under investigation, the physician is left with four questions.

- Is ADAM suspected (Figure 2)?

- Is the history consistent with symptoms of both ED and ADAM (Table 1)?

- Is ED the major concern? If yes, proceed directly to the flow chart in Figure 1.

- Is sexual desire the major concern? If yes, proceed directly to the investigation algorithm in Figure 2.

In contrast to the algorithmic approach to ADAM, ED has a variety of management steps and options. Over the past decade, the approach to the man with ED has become simplified with the advent of oral agents. A patient-directed approach is generally advised, allowing the patient and his partner to guide the choice of treatment. Important research into the underlying causes of ED has led to innovative and minimally invasive diagnostic approaches. In truth, however, the era of directed therapy, in which a specific deficit in the normal cascade of erectile function is definable, 26 . has not yet begun. 
At present, in the majority of men with ED, a careful history is taken, in addition to a limited physical examination; a hormonal screen is completed in cases in which a desire deficit is encountered. When the results of this screen are normal, little further workup into the possible hormonal impact on sexual function is usually recommended. Treatment options for ED are generally presented from least to most invasive, and a therapeutic trial is undertaken. From a practical standpoint, in the vast percentage of men, this means that an oral agent is prescribed, unless specific contraindications exist (eg, nitrate use). In most men, this approach avoids an extensive and expensive workup, and restores adequate erections for sexual function. In cases in which failure of this first-line treatment is encountered, consideration of intraurethral, intracorporal or vacuum devices is generally suggested for second-line treatments. In circumstances in which second-line treatments are unacceptable or fail, surgical prosthesis implants are a useful permanent solution.

In some cases, initial management of the patient presenting with ED and a significant desire deficit may not cleanly fit any of the above treatment algorithms. If both ED and desire deficits exist, a trial of testosterone supplementation may effectively treat both issues. In cases in which there is a clear predominance of the ED, success with hormone supplements alone rarely is successful, in the author's experience.

\section{CONCLUSIONS}

Although quite distinct in their underlying pathophysiological basis, ED and ADAM share many presenting symptoms and commonly cause diagnostic confusion. Focusing on the central issue of sexual interest and obtaining a clear sexual history from the patient that defines the degree of erectile function usually defines which entity is the cause of sexual dysfunction.

Asking men older than 50 years about their sexual health is important (16). Therapeutic options are available and can effectively restore sexual function to most of the men affected by these symptoms, whether they are of vascular or hor- monal origin. Providing the patient with a forum in which sex can be discussed is often the most important therapeutic step in treating this large cohort of aging men.

\section{REFERENCES}

1. Lue TF. Erectile dysfunction. N Engl J Med 2000;342:1802-13.

2. Kuthe A, Wiedenroth A, Magert HJ, et al. Expression of different phosphodiesterase genes in human cavernous smooth muscle. J Urol 2001;165:280-3.

3. Brock GB. New Horizons in Erectile Dysfunction Therapy. <http://www.medscape.com/Medscape/urology/ TreatmentUpdate/2001/tu01/public/toc-tu01.html> (Version current at September 12,2001)

4. Feldman HA, Goldstein I, Hatzichriston DG, et al. Impotence and its medical and psychosocial correlates: results of the Massachusetts Male Aging Study. J Urol 1994;151:54-61.

5. Johannes $\mathrm{CB}$, Araujo AB, Feldman HA, et al. Incidence of $\mathrm{ED}$ in men 40 to 69 years old: longitudinal results from the Massachusetts Male Aging Study. J Urol 2000;163:460-3.

6. Burchardt M, Burchardt T, Baer L, et al. Hypertension is associated with severe ED. J Urol 2000;164:1188-91.

7. Goldstein I, Lue TF, Padma-Nathan H, et al. Oral sildenafil in the treatment of ED. N Engl J Med 1998;338:1397-404.

8. Giuliano F, Montorsi F, Mirone V, et al. The Sildenafil Multicenter Study Group. Switching from intracavernous prostaglandin E1 injections to oral sildenafil citrate in patients with ED: results of a multicenter European study. J Urol 2000;164:708-11.

9. Christiansen E, Guirguis WR, Cox D, et al. Long-term efficacy and safety of oral Viagra (sildenafil citrate) in men with erectile dysfunction and the effect of randomized treatment withdrawal. Int J Impot Res 2000;12:177-82.

10. Morales A, Heaton JPW, Carson CC. Andropause: A misnomer for a true clinical entity. J Urol 2001;163:705-12.

11. Bain J. Andropause. Testosterone replacement therapy for aging men. Can Fam Physician 2001;47:91-7.

12.Tremblay RR, Morales A. Canadian practice recommendations for screening, monitoring and treating men affected by andropause or partial androgen deficiency. Aging Male 1998;1:213-8.

13. Gooren LI. The age-related decline in androgen levels in men: clinically significant? Br J Urol 1996;78:763-8.

14. Comhaire FH. Andropause: hormone replacement therapy in the ageing male. Eur Urol 2000;38:655-62.

15. Vermeulen A, Kaufman JM. Ageing of the hypothalmopituitary-testicular axis in men. Horm Res 1995;43:25-8.

16. Nieschlag E, Lammers U, Freischem CW, Langer K, Wicking EJ. Reproductive function in young fathers and grandfathers. J Clin Endocrinol Metab 1982;55:676-81.

\footnotetext{
A note from the Editor-in-chief

Where does the discussion of lifestyle factors belong in the treatment algorithm? For example, exercise is a well accepted prescription for most coronary patients, a group that shares many of the same risk factors as the populations with androgen deficiency in the aging male and erectile dysfunction. It should be considered for these patients as well.
} 